\title{
De novo CNV analysis implicates specific abnormalities of postsynaptic signalling complexes in the pathogenesis of schizophrenia
}

\author{
G Kirov ${ }^{1}$, AJ Pocklington ${ }^{1}$, P Holmans ${ }^{1}$, D Ivanov ${ }^{1}$, M Ikeda $^{2}$, D Ruderfer ${ }^{3,4,5}$, J Moran ${ }^{5}$, K Chambert ${ }^{5}$,
} D Toncheva ${ }^{6}$, L Georgieva ${ }^{1}$, D Grozeva ${ }^{1}$, M Fjodorova ${ }^{1}$, R Wollerton ${ }^{1}$, E Rees ${ }^{1}$, I Nikolov ${ }^{1}$, LN van de Lagemaat ${ }^{7}$, À Bayés ${ }^{7}, E$ Fernandez ${ }^{8}$, PI Olason ${ }^{9}$, Y Böttcher ${ }^{9}$, NH Komiyama ${ }^{7}$, MO Collins ${ }^{10}$, J Choudhary ${ }^{10}$, K Stefansson ${ }^{9}$, H Stefansson $^{9}$, SGN Grant ${ }^{7}$, S Purcell ${ }^{3,4,5}$, P Sklar ${ }^{3,4,5}$, MC O'Donovan ${ }^{1}$ and MJ Owen ${ }^{1}$

\begin{abstract}
${ }^{1}$ Department of Psychological Medicine and Neurology, MRC Centre for Neuropsychiatric Genetics and Genomics, School of Medicine, Neuroscience and Mental Health Research Institute, Cardiff University, Cardiff, UK; ${ }^{2}$ Department of Psychiatry, School of Medicine, Fujita Health University, Toyoake, Aichi, Japan; ${ }^{3}$ Department of Psychiatry, Psychiatric and Neurodevelopmental Genetics Unit, Massachusetts General Hospital and Harvard Medical School, Boston, MA, USA; ${ }^{4}$ Center for Human Genetics Research, Massachusetts General Hospital, Boston, MA, USA; ${ }^{5}$ Stanley Center for Psychiatric Research, Broad Institute, Cambridge, MA, USA; ${ }^{6}$ University Hospital Maichin Dom, Sofia, Bulgaria; ${ }^{7}$ Genes to Cognition Program, School of Molecular and Clinical Medicine, Edinburgh University, Edinburgh, UK; ${ }^{8}$ VIB Department of Molecular and Developmental Genetics, KU Leuven Medical School, Leuven, Belgium; ${ }^{9}$ deCODE Genetics, Reykjavík, Iceland and ${ }^{10}$ The Wellcome Trust Sanger Institute, Hinxton, Cambridgeshire, UK
\end{abstract}

\begin{abstract}
A small number of rare, recurrent genomic copy number variants (CNVs) are known to substantially increase susceptibility to schizophrenia. As a consequence of the low fecundity in people with schizophrenia and other neurodevelopmental phenotypes to which these CNVs contribute, CNVs with large effects on risk are likely to be rapidly removed from the population by natural selection. Accordingly, such CNVs must frequently occur as recurrent de novo mutations. In a sample of 662 schizophrenia proband-parent trios, we found that rare de novo CNV mutations were significantly more frequent in cases $(5.1 \%$ all cases, $5.5 \%$ family history negative) compared with $2.2 \%$ among 2623 controls, confirming the involvement of de novo CNVs in the pathogenesis of schizophrenia. Eight de novo CNVs occurred at four known schizophrenia loci (3q29, 15q11.2, 15q13.3 and 16p11.2). De novo CNVs of known pathogenic significance in other genomic disorders were also observed, including deletion at the TAR (thrombocytopenia absent radius) region on 1q21.1 and duplication at the WBS (WilliamsBeuren syndrome) region at $7 q 11.23$. Multiple de novos spanned genes encoding members of the DLG (discs large) family of membrane-associated guanylate kinases (MAGUKs) that are components of the postsynaptic density (PSD). Two de novos also affected EHMT1, a histone methyl transferase known to directly regulate $D L G$ family members. Using a systems biology approach and merging novel CNV and proteomics data sets, systematic analysis of synaptic protein complexes showed that, compared with control CNVs, case de novos were significantly enriched for the PSD proteome $\left(P=1.72 \times 10^{-6}\right)$. This was largely explained by enrichment for members of the $N$-methyl-D-aspartate receptor (NMDAR) $\left(P=4.24 \times 10^{-6}\right)$ and neuronal activity-regulated cytoskeleton-associated protein (ARC) $\left(P=3.78 \times 10^{-8}\right)$ postsynaptic signalling complexes. In an analysis of 18492 subjects (7907 cases and 10585 controls), case CNVs were enriched for members of the NMDAR complex $(P=0.0015)$ but not ARC $(P=0.14)$. Our data indicate that defects in NMDAR postsynaptic signalling and, possibly, ARC complexes, which are known to be important in synaptic plasticity and cognition, play a significant role in the pathogenesis of schizophrenia.

Molecular Psychiatry (2012) 17, 142-153; doi:10.1038/mp.2011.154; published online 15 November 2011
\end{abstract}

Keywords: CNV; de novo; DLG; EHMT1; postsynaptic; schizophrenia

Correspondence: Dr G Kirov, PhD, MRCPsych or Professor MC O'Donovan, PhD, FRCPsych, Department of Psychological Medicine and Neurology, Henry Wellcome Building, School of Medicine, Cardiff University, Heath Park, Cardiff, CF14 4XN, UK. E-mail: kirov@cardiff.ac.uk or odonovanmc@cf.ac.uk

Received 27 July 2011; revised 28 September 2011; accepted 4 October 2011; published online 15 November 2011
Introduction

Genome-wide association studies have found strong evidence for association between schizophrenia and a number of genetic variants, both common and rare. ${ }^{1}$ So far, the evidence for rare variants comes mainly 
from the analysis of deletions and duplications of segments of DNA known as copy number variants (CNVs). Cumulatively, as a general class, large $(>100 \mathrm{~kb})$ rare $(<1 \%)$ CNVs occur more frequently in those with schizophrenia ${ }^{2,3}$ than controls, and several individual CNV loci have been strongly implicated as risk factors for schizophrenia with high degrees of statistical confidence. These include deletions at 1q21.1, NRXN1, 3q29, 15q11.2, 15q13.3, 22q11.2 and duplications at VIPR2, 16p11.2, 16p13.1 and 15q11-q13. ${ }^{2,4-13}$ Pleiotropic effects are common, the same CNV often conferring risk for a range of neurodevelopmental phenotypes including autism, mental retardation, attention deficit hyperactivity disorder and epilepsy, although interestingly, and in contrast to the findings with common risk alleles, there is little evidence that schizophrenia-associated CNVs confer risk for bipolar disorder. ${ }^{14}$

All of the currently known risk CNVs are rare (control frequencies typically <0.001) and confer substantial effects on risk (odds ratios 3-30). The known risk CNVs occur in $2-3 \%$ of cases, but it is likely that many other risk CNV loci remain to be identified. Most schizophrenia-associated CNVs span multiple genes, limiting our ability to make strong inferences regarding pathogenesis. Important exceptions are deletions of NRXN1, encoding the presynaptic neuronal cell adhesion molecule neurexin $1,{ }^{11,15}$ pointing to the importance of as yet unspecified abnormalities of synaptic function in the disorder. Also, obscuring mechanistic insights from the CNV data are that most reported CNVs occurring in cases are too rare to allow clear demonstration of association statistically. One way to circumvent this is to test whether particular functionally related groups or sets of genes are enriched among case CNVs, rather than trying to interpret the results from individual CNVs. A limitation of this approach is that the enrichment of CNVs seen in case-control studies is modest; ${ }^{2}$ indeed, one large study has reported no overall excess of CNVs in cases at all. ${ }^{4}$ This implies that among sets of CNVs drawn from cases, only a small proportion can be expected to be true risk factors for the disorder. Nevertheless, gene-set enrichment studies have supported conclusions drawn from consideration of genes affected by individual CNVs in schizophrenia ${ }^{16}$ by observing enrichment in schizophrenia of genes involved in a range of brain functions, for example, those encoding products involved in nitric oxide signalling, synaptic long-term potentiation and glutamate receptor signalling, ${ }^{17}$ or genes in a broad category corresponding to the gene ontology (GO) category 'synaptic transmission'. ${ }^{18}$ However, it has been noted that the early gene-set studies did not allow for important confounders, in particular the large size of genes implicated in brain function, and that the conclusions that can be drawn are consequently unclear. ${ }^{19}$

Schizophrenia is associated with reduced fecundity, $\sim 40 \%$ that of the general population, ${ }^{20}$ or even lower according to the largest population-based

study. ${ }^{21}$ It follows that schizophrenia-related mutations of large effect should be rare because of intense purifying selection, and those that occur in multiple unrelated individuals are likely to do so through independent de novo mutations., ${ }^{72-24}$ One study on de novo $\mathrm{CNV}$ mutation in schizophrenia ${ }^{24}$ showed that the rate of de novo CNV mutation in probands with no family history was 8 times higher in cases than in controls. This marked elevation in the rate of de novo CNVs contrasts with the relatively modest elevation in the rate of CNVs seen in case-control studies, ${ }^{2,4}$ and suggests that sets of de novos might be more informative for gene-set enrichment analyses.

Here, we report the largest analysis of de novo CNVs in schizophrenia to date. Our aims were to identify novel CNVs that increase risk of schizophrenia and to illuminate aspects of the pathophysiology of the disorder through gene-set enrichment analyses informed by recently curated proteomics data sets of synaptic protein complexes.

\section{Materials and methods}

\section{Samples}

Bulgaria The sample for de novo CNV analysis comprised 662 Bulgarian parent-proband trios from 638 families. We did not exclude probands $(N=61)$ with a history of psychosis in a parent as none of the risk CNVs identified to date are sufficiently penetrant to fully explain the disorder in carriers. All cases had been hospitalised and met DSM-IV (Diagnostic and Statistical Manual of Mental Disorders-fourth edition) criteria for schizophrenia or schizoaffective disorder based upon SCAN (Schedules for Clinical Assessment in Neuropsychiatry) interview by psychiatrists, and review of case notes. Cases were recruited from general adult psychiatric services and were typical of those attending those services. Although they did not have formal IQ assessments, all attended mainstream schools from which people with known mental retardation were excluded. All participants provided informed consent. Further details concerning ascertainment and diagnostic practices are provided in the Supplementary Material. All DNA samples were derived from peripheral venous blood.

Icelandic control de novos deCODE Genetics provided data for 2623 complete parent-offspring trios from the Icelandic population. ${ }^{7}$ Probands known to be affected with neurodevelopmental/psychiatric disorders (schizophrenia, autism, attention deficit hyperactivity disorder, mental retardation and bipolar affective disorder) had been excluded.

Autism case and control de novos Data on de novo rates in autism cases and their unaffected siblings are directly taken from the recent large study of Sanders et al. ${ }^{25}$ based upon the Illumina (San Diego, CA, USA) $1 \mathrm{M}$ high-density array. 
Case-control data sets We used four large publicly available data sets to which we also had access to the raw data. (1) The International Schizophrenia Consortium (ISC), ${ }^{2}$ which included 3391 cases and 3181 controls genotyped with Affymetrix 6.0 or 5.0 arrays (Affymetrix, Santa Clara, CA, USA). Note that 328 Bulgarian cases from that study are probands in our trios (although their parents were not genotyped for de novo calling in the ISC study). We excluded those subjects from the ISC data. The ISC also included 605 unrelated controls recruited by us in Bulgaria (details in ref. 2) and those publicly available data were included in the present study. (2) The Molecular Genetics of Schizophrenia (MGS) Consortium, ${ }^{4}$ which included 3192 cases and 3437 controls genotyped with Affymetrix 6.0 arrays. (3) A UK case-control study of 471 schizophrenia and 2792 controls genotyped using the Affymetrix (Affymetrix) GeneChip500K Mapping Array (see ref. 3 for details of the sample and CNV calling). (4) CNV data reported by Ikeda et al. ${ }^{26}$ comprising a Japanese sample of 519 cases and 513 controls. Including the data from the current study on transmissions and non-transmissions to affected offspring, and excluding the 328 overlapping Bulgarian cases, the combined case-control data sets contain a total of 7907 independent cases and 10585 controls.

\section{Genotyping and CNV analysis}

Bulgarian samples Full details are provided in the Supplementary Material (Sections 1-3). All participants were genotyped with Affymetrix 6.0 arrays (Affymetrix) at the Broad Institute of Harvard and Massachusetts Institute of Technology. As an initial screen, we used Genotyping Console 4.0 software (Affymetrix, Santa Clara, CA, USA) to call autosomal CNVs, restricting initial calls to $\geqslant 10 \mathrm{~kb}$ and $\geqslant 10$ probes. We next excluded individuals with >50 CNV calls, as these were outliers from the distribution, followed by CNV loci with a frequency $>1 \%$ in the whole sample. We then excluded putative CNVs $<15 \mathrm{~kb}$, covered by $<15$ probes, or where $>50 \%$ of their length overlapped low copy repeats. Calls compatible with a de novo were made if a proband CNV was not spanned $>50 \%$ of its length by a CNV in either parent. Probands who had large numbers of apparent de novos (>10) were excluded. After this initial screen with relaxed criteria to capture as many potential de novos as possible, we measured probe Log2 ratios derived from PennCNV. ${ }^{27}$ We then used a slight modification of the MeZOD algorithm $^{12}$ (Supplementary Section 3) to visualise outlier signals in probands potentially indicative of de novos (Figure 1). Again, we used relaxed criteria, only excluding clear false positives (Supplementary Section 3). For those whose patterns were either highly suggestive of a de novo $(N=40$, Figure 1a) or were ambiguous $(N=33$, Figure $1 \mathrm{~b})$, proband and parent DNAs were examined on custom Agilent SurePrint G3 Human CGH Microarrays on which 50-200 probes were placed to cover each CNV
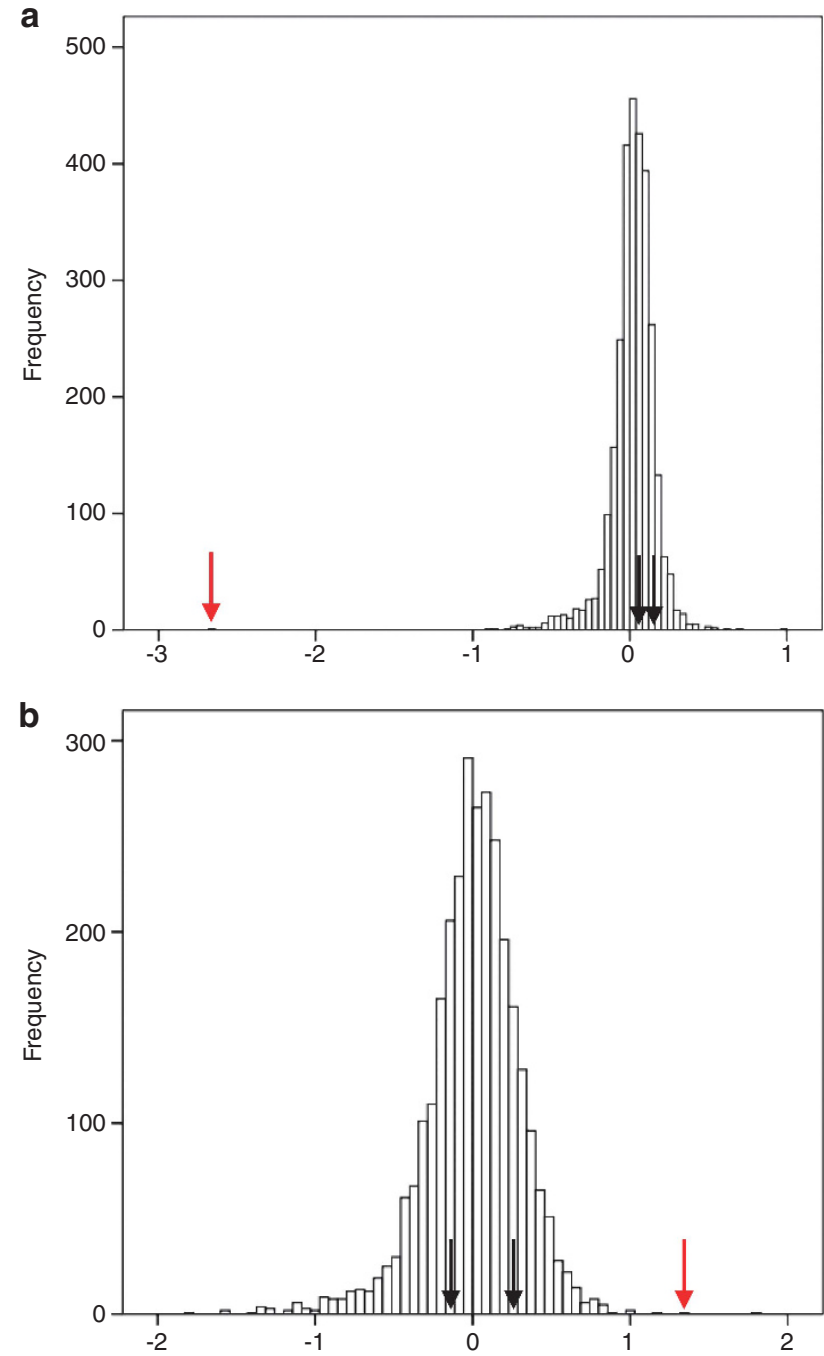

Figure 1 Histograms of distributions of $z$-scores. (a) A suggestive de novo and (b) an ambiguous de novo MeZOD call. Black arrows indicate the position of a parent, and red arrows of a child. The $x$ axis shows the median $z$-scores for all individuals for a particular copy number variant (CNV) region.

(depending on CNV size). For quality control purposes, we also included probes on all putative de novos identified by the first-pass Genotyping Console analysis, but that were subsequently rejected as false positives by the MeZOD method.

To re-call CNVs in the Bulgarian controls, we used the same filtering criteria and accepted only those considered highly suggestive by the MeZOD. Although we did not validate these on Agilent arrays, our calls have a demonstrable low false positive rate $(<1 \%$, Supplementary Section 4$)$. This is much less than the corresponding false positive rate for de novos whose rarity confers more unfavourable signal-to-noise characteristics.

Icelandic samples These were genotyped using Illumina bead arrays (HumanHap317, HumanHap370 and HumanHap1M). BeadStudio (Illumina, San Diego, 
CA, USA; version 2.0) was used to call genotypes, normalise signal intensity data and establish the log $\mathrm{R}$ ratio and B allele frequency at every single-nucleotide polymorphism. Samples passing quality control were examined using PennCNV (10.1101/gr.6861907). Calls required 10 consecutive markers based upon the subset of markers present on all genotyping chips listed above (the HumanHap317 content). All putative de novo events were visually inspected using DosageMiner software (developed by deCODE Genetics). CNVs were excluded according to low copy repeat content and frequency as for the Bulgarian sample. This resulted in $59 \mathrm{CNVs}$, an autosomal de novo rate of $2.2 \%$. Given the difference in the platforms, we undertook a number of analyses to confirm that the Icelandic de novos are a suitable comparator group for the case de novos (see Results and Supplementary Material).

MGS/ISC/UK/Japan. MGS samples were analysed in the same way as the Bulgarian samples including MeZOD. Data for ISC $^{2}$ UK $^{3}$ and Japanese samples ${ }^{26}$ were taken from the original publications, and CNVs at loci of interest were manually verified in the available raw data (further information in Supplementary Material).

\section{Gene set analyses}

Sets. We collated experimentally defined proteomic data sets corresponding to the structures listed in Table 2. The details of how those gene sets were collated are provided in Supplementary Section 10. We also examined sets based upon the Gene Ontology system (GO sets) in the gene2go file available from the NCBI (National Center for Biotechnology Information) on 28 July 2010 (Supplementary Section 11).

Statistical approaches. A gene was considered 'hit' if a CNV was overlapped according to the NCBI Build 36.3. Full details of mapping are given as Supplementary Section 10.

The impact of biases relating to gene-set analyses of CNVs have been discussed elsewhere. ${ }^{19}$ To overcome those biases, we fitted the following logistic regression models to the combined set of case and control (or control de novo) CNVs and compared the change in deviance between (1) and (2).

(1) logit $(\operatorname{pr}($ case $))=\mathrm{CNV}$ size + Total number of genes hit outside the gene set + number of genes hit in the gene set.

(2) $\operatorname{logit}($ pr $($ case $))=\mathrm{CNV}$ size + Total number of genes hit outside the gene set.

Significance was assessed by one-sided test of an excess of genes hit in the gene set by case CNVs. The inclusion of CNV size allows for case de novo CNVs being larger than typical CNVs (and thus likely to hit more genes). Inclusion of the total number of genes hit outside the gene set in the regression corrects for case CNVs hitting more genes overall (regardless of function) than control CNVs. Although explicitly adjusted for in the above analysis, to confirm that

the results are not due to the fact that de novo CNVs are more likely to hit genes, we also performed an analysis restricted to CNVs that hit genes.

We used the same method to compare the number of genes in gene sets hit by case de novos with those hit by (1) 1367 CNVs from the 605 Bulgarian unaffected controls (2) 59 de novos found in Icelandic controls and (3) 14 control de novos from the unaffected sibs of autism probands. ${ }^{25}$ The analyses control for different sources of potential bias including array type (the Bulgarian controls) and the possibility that de novos have fundamentally different characteristics (other than size that is adjusted for) than control CNVs.

To investigate the impact of using 'control' CNVs, we undertook a random placement analysis comparing the number of de novo CNVs hitting each gene set with that found when CNV locations were randomised, importantly ensuring that each random assignment hits at least one gene, and that the probability of a gene being hit was proportional to its length (Supplementary Section 12).

Partitioning the signals in gene sets. Gene sets are not fully independent, for example, some members of the synaptic vesicle set (Table 2) are also members of the postsynaptic density (PSD). To determine which among overlapping sets appeared to be responsible for a geneset enrichment, we undertook conditional regression analyses as described in Supplementary Section 13.

Meta-analysis of case-controls. For meta-analysis combining cases and controls from multiple studies, we included in the above regression models a "study" term added as an $N$-level factor (where $N=$ number of case/control sets being combined). This makes the analysis robust to differences between studies in chip, analytic method and other study-specific factors.

\section{Results}

We identified 34 confirmed de novo CNVs (Table 1), a rate in all cases of $5.1 \%$. Detailed descriptions of individual de novo CNVs are given in Supplementary Section 6 and in the Discussion section. As in an earlier study, ${ }^{24}$ the de novo rate in those with a history of psychosis in a parent was lower $(1.6 \%)$ than in those without such history (5.5\%), although this was not statistically significant. Parents of probands with de novos were not older at the time of birth of their children than parents of probands without de novos: ( 27.8 vs 28.7 years, respectively, for fathers and 25.1 vs 25.1 years for mothers). Probands with de novo CNVs (Table 1) did not differ from the rest of the probands regarding age at onset (23.9 vs 23.8 years, $P=0.9$ ) and average school results ( 4.5 vs $4.7, P=0.5$ ), and both sets of probands had similar numbers of children (0.52 vs $0.59, P=0.6)$. In those instances (21) where it was possible to determine the parental origin, more de novos occurred in the paternal $(P=14)$ than the maternal $(n=7)$ genome but this 


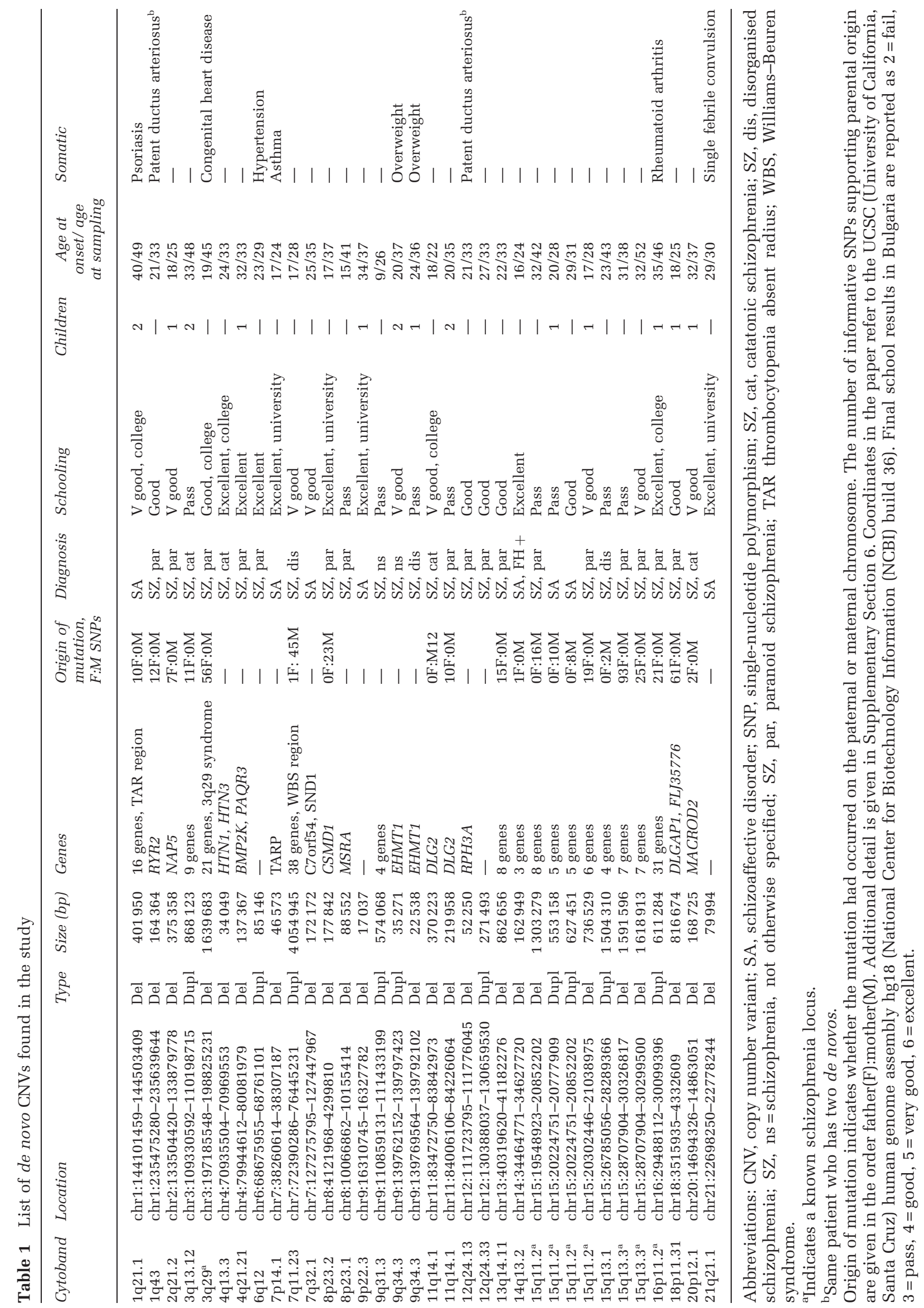


was not statistically significant $(P=0.13)$. The nonsignificant excess of paternal de novos was largely attributable to CNVs that were not generated by nonallelic homologous recombination, eight such events being observed on chromosomes of paternal origin compared with two on those that were maternally derived, although this is not significantly different from chance $(P=0.06)$.

In order to estimate the de novo $\mathrm{CNV}$ rate in controls for comparison with cases, we compared the case de novo rate with that in controls from two sources, the Icelandic population controls and the unaffected sibs from a recent large study of autism ${ }^{25}$ (Supplementary Table S3). The de novo mutation rate in our cases was higher than in both sets of controls (2.2\%, $P=0.00015$ and $1.6 \%, P=0.00008$, respectively), both of which had a similar rate $(P=0.28)$ despite differences in the density of markers in the control genotyping platforms.

In order to exclude the possibility that the increased de novo rate seen in cases reflected the different platforms ${ }^{28}$ used in our cases and the control studies, we undertook sensitivity analyses. If the elevation in de novos in cases is an artefact of greater call sensitivity in the present study, the enrichment we observed should be biased towards smaller CNVs, larger CNVs being called reliably after exclusion of CNVs spanning complex repeat regions (as we have done). ${ }^{28}$ However, relative enrichment for de novos among cases was similar for large de novos $>500 \mathrm{~kb}$ $(2.1 \%$ vs $0.8 \%, P=0.0014)$ as it was for small de novos $<200 \mathrm{~kb}(2.3 \%$ vs $0.9 \%, P=0.0035)$, and the overall size distribution of case de novos was not shifted towards smaller CNVs (Figure 2 and Supplementary Table S3) compared with the control de novos. In general, duplications are less easily detected by microarrays than deletions. To exclude the possibility that the excess of de novos in cases

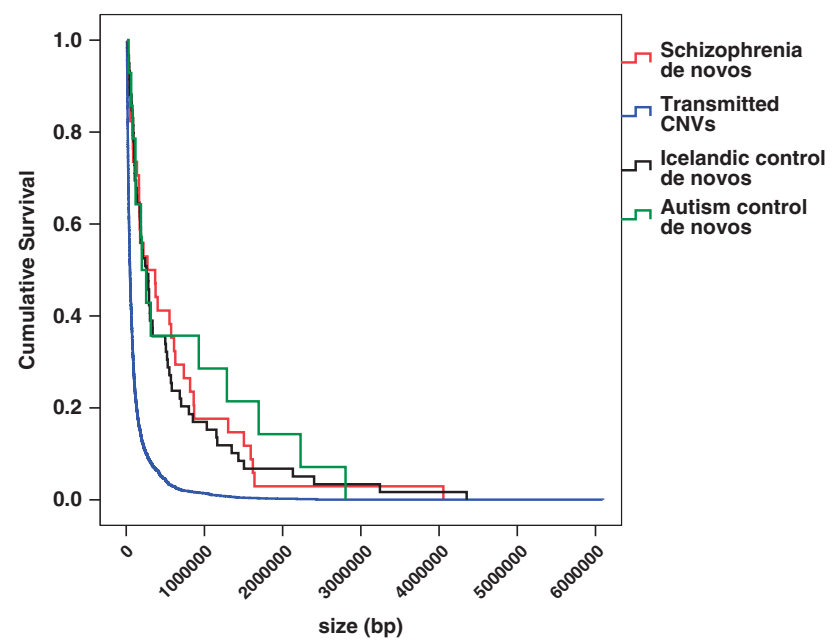

Figure 2 Size of copy number variants (CNVs). KaplanMeier survival graph for the size of de novo CNVs in cases, Icelandic controls, Bulgarian controls and unaffected siblings of autism probands. ${ }^{25}$ reflects a lower sensitivity of the control platforms to detect duplications, we also examined the duplication/deletion ratios in the data sets. These were not significantly different $(P>0.35$ for each sample), although contrary to the hypothesis of a selective loss of sensitivity to detect duplications, both sets of controls actually had a higher proportion of duplications (Icelandic $=0.39$, Autism controls $=0.36$ ) than the cases (0.29). Further details on the size distribution of the de novo CNV are given in Supplementary Section 8, and of the full sensitivity analyses in Supplementary Section 1. Finally, we note that the control rates were similar to those in our experimental group with an affected parent (who according to an earlier work ${ }^{24}$ do not have elevated rates of de novo mutation), suggesting that technical variation between our own and other studies does not make a major impact on our conclusions.

\section{Analysis of de novo loci in case-control studies}

We examined the fully independent case-control data sets for rare CNVs at the novel loci affected by case de novos, including CNVs only of the same class that had been observed to have occurred as de novos (that is, deletions, duplications or both where relevant) (Supplementary Section 7) that intersected at least one exon of a gene (details in Supplementary Table S1). Even after an extremely conservative approach of excluding all CNVs (deletions and duplications) at known schizophrenia loci represented among our de novos (3q29, 15q11.2, 15q13.3 and 16p11.2), we found rates of $0.4 \%(32 / 7907)$ in cases and $0.21 \%(22 / 10585)$ in controls, a twofold enrichment (Fisher one-tailed $P=0.012$ ). We did not obtain evidence for association to individual CNV loci at a level that would survive correction for multiple testing $(N=19$ excluding the known schizophrenia loci, giving a Bonferroni corrected threshold of $P=0.0025)$. However, nominally significant associations ( $P$ uncorrected <0.05) were observed for deletions at DLG2 $(P=0.02)$ and MSRA $(P=0.03)$, whereas the EHMT1 locus just failed to reach this uncorrected threshold $(P=0.055)$. Of interest, although not even nominally significant, we also observed an excess of CNVs in cases at two other loci known to be implicated in nonpsychiatric genomic disorders: deletions of the TAR (thrombocytopenia absent radius) region $(P=0.11)$ and duplications of the WBS (Williams-Beuren syndrome) region $(P=0.11)$.

\section{Gene-set analyses}

We initially undertook gene-set analyses based upon proteomics-based annotations (Table 2 and Supplementary Section 11). To avoid multiple testing involved in subgroup analysis, we present the findings for the full sample of de novos, although we note that exclusion of the single de novo in a proband with a family history of psychosis in a parent made essentially no difference to the results. Compared with Bulgarian control CNVs, we found a highly 
Table 2 Enrichment of gene sets for de novo CNV hits in comparison with control CNVs

\begin{tabular}{|c|c|c|c|c|c|c|}
\hline \multirow[t]{2}{*}{ Gene set } & \multirow[t]{2}{*}{$\mathrm{N}$ genes } & \multicolumn{4}{|c|}{$\mathrm{N}$ genes hit by CNVs P-value } & \multirow[t]{2}{*}{ Genes hit by $S C Z$ de novos } \\
\hline & & $\begin{array}{c}S C Z \\
\text { de novo } \\
(34)\end{array}$ & $\begin{array}{c}\text { Bulgarian } \\
\text { control } \\
(1367)\end{array}$ & $\begin{array}{c}\text { Icelandic } \\
\text { control } \\
\text { de novo (59) }\end{array}$ & $\begin{array}{c}\text { Autism } \\
\text { control de } \\
\text { novo (14) }\end{array}$ & \\
\hline PSD & 664 & 19 & $49\left(\mathbf{1 . 7 2} \times \mathbf{1 0}^{-\mathbf{6}}\right)$ & $13(\mathbf{0 . 0 4 5 )}$ & $4(0.11)$ & $\begin{array}{l}D L G 1, D L G 2, D L G A P 1, R Y R 2, S N D 1, \text { STX1A, } \\
\text { MDH2, HSPB1, YWHAG, RPH3A, CYFIP1, } \\
\text { TJP1, ALDOA, TAOK2, MAPK3 }\end{array}$ \\
\hline ARC complex & 25 & 8 & $7\left(3.78 \times 10^{-8}\right)$ & $1\left(2.51 \times 10^{-4}\right)$ & $0(\mathbf{0 . 0 0 4 9 )}$ & $D L G 1, D L G 2, D L G A P 1, C Y F I P 1$ \\
\hline NMDAR complex & 59 & 8 & $6\left(4.24 \times 10^{-6}\right)$ & $2(\mathbf{0 . 0 0 6 1})$ & $0(\mathbf{0 . 0 1})$ & $\begin{array}{l}D L G 1, D L G 2, D L G A P 1, S T X 1 A, Y W H A G \\
\text { TJP1, MAPK3 }\end{array}$ \\
\hline PSD-95 complex & 58 & 4 & $3\left(1.17 \times 10^{-5}\right)$ & $1(\mathbf{0 . 0 1 7})$ & $0(\mathbf{0 . 0 3 3})$ & $D L G 1, D L G 2, D L G A P 1$ \\
\hline mGluR5 complex & 37 & 3 & $4(\mathbf{0 . 0 2 6})$ & $2(0.45)$ & $0(0.15)$ & $Y W H A G, R P H 3 A, A L D O A$ \\
\hline Presynapse & 426 & 8 & $25(\mathbf{0 . 0 3 3 )}$ & $8(0.32)$ & $2(0.28)$ & STX1A, RPH3A, CYFIP1, ALDOA, MDH2 \\
\hline Synaptic vesicle & 333 & 7 & $20(\mathbf{0 . 0 1 4})$ & $8(0.39)$ & $2(0.31)$ & STX1A, RPH3A, CYFIP1, ALDOA \\
\hline Active zone & 176 & 2 & $6(0.29)$ & $3(0.91)$ & $0(0.26)$ & $A L D O A, M D H 2$ \\
\hline Nucleus & 160 & 5 & $10(\mathbf{0 . 0 0 2 4})$ & $2(\mathbf{0 . 0 2 6})$ & $0(\mathbf{0 . 0 1 8})$ & CYFIP1, TJP1 \\
\hline Mitochondrion & 189 & 3 & $9(0.41)$ & $1(0.11)$ & $0(0.093)$ & МDH2, BDH1, KIAA0564 \\
\hline Cytoplasm & 263 & 4 & $11(0.68)$ & $3(0.55)$ & $0(0.15)$ & EIF4H, YWHAG, MSRA, MVP \\
\hline Endoplasmic reticulum & 94 & 1 & $3(0.75)$ & $0(0.18)$ & $0(0.31)$ & POR \\
\hline $\begin{array}{l}\text { Endoplasmic reticulum/ } \\
\text { Golgi-derived vesicles }\end{array}$ & 94 & 0 & 0 & 0 & 0 & \\
\hline Recycling endosomes & 65 & 0 & $2(0.83)$ & 0 & 0 & \\
\hline Early endosomes & 17 & 0 & $1(0.82)$ & 0 & 0 & \\
\hline Golgi & 31 & 0 & $1(0.82)$ & 0 & 0 & \\
\hline Plasma membrane & 50 & 0 & $2(0.61)$ & 0 & 0 & \\
\hline
\end{tabular}

Abbreviations: ARC, activity-regulated cytoskeleton-associated protein; CNV, copy number variant; NMDAR, N-methyl-Daspartate receptor; PSD, postsynaptic density; SCZ, schizophrenia.

Gene sets were tested for enrichment in 34 schizophrenia ('SCZ') de novo CNVs compared with 1367 CNVs found in 605 Bulgarian controls ('controls'), 59 de novo CNVs found in 2623 unaffected individuals from the Icelandic population ('Icelandic control de novo') and 14 de novo CNVs found in unaffected siblings of autism proband from the study by Sanders et al. ${ }^{25}$ ('Autism control de novo'). ' $N$ genes' refers to number of genes in the set. $P$-values are presented underneath the number of genes hit and correspond to one-tailed tests of an excess of gene hits in case CNVs. $P$-values in bold are significant. ' $N$ genes hit by CNVs' refers to the number of times any gene in the set is hit by a CNV. The unique genes hit in each set are given in the final column. Genes in bold are present in multiple subcellular components.

significant excess of PSD genes within case de novos $\left(P=1.72 \times 10^{-6}\right.$; Table 2). As expected, the results where the analysis was restricted to CNVs hitting genes were similar to those of the primary analysis (data not shown).

Significant enrichments were also observed in presynaptic vesicle and nuclear gene sets, but not after conditioning on the PSD $\left(P_{\min }=0.66\right)$, whereas the PSD gene set remained significantly enriched for hits after conditioning on the other sets individually $\left(P_{\max }=5.20 \times 10^{-3}\right)$ or combined $(P=0.016)$ (Supplementary Section 13). The most parsimonious interpretation is that our findings specifically implicate the PSD, although we cannot exclude the possibility of effects across multiple functional sets.

To explore our findings in the context of a less restricted set of classifications, we performed enrichment analyses using the GO annotation. Of all categories, 'the synapse' (GO: 45202) was by two orders of magnitude the most significantly enriched $\left(P=9.6 \times 10^{-9}\right) \quad($ Supplementary Section 11 and Supplementary Table S4) but most of this signal was attributable to the PSD gene set $(P=0.049$ after removing PSD genes, Supplementary Table S5). Only one subcategory of 'the synapse' GO: 45202 was enriched after PSD genes were removed: GO: 30672 'synaptic vesicle membrane' $(P=0.036$, Supplementary Table S5).

Aiming to localise more specifically the source of the PSD gene-set enrichment, we tested gene sets encoding PSD components (Table 2 and Figure 3). Activity-regulated cytoskeleton-associated protein (ARC; $P=3.78 \times 10^{-8}$ ), $N$-methyl-D-aspartate receptor (NMDAR; $P=4.24 \times 10^{-6}$ ) and PSD-95 complex $\left(P=1.17 \times 10^{-5}\right)$ genes were highly significantly enriched among the de novos. However, conditional analyses revealed that the relatively small ARC and NMDAR sets explained both the PSD (conditional $\mathrm{P}_{\mathrm{PSD}}=0.231$ ) and PSD-95 (conditional $\mathrm{P}_{\mathrm{PSD}-95}=0.603$ ) enrichments but that the enrichments in ARC and NMDAR were partially independent of each other (conditional $P=2.17 \times 10^{-4}$ and $P=0.019$, respectively) (Supplementary Section 13). ARC and NMDAR sets also explained most of the enrichment for de novos in 'the synapse', this GO category being only marginally enriched $(P=0.017)$ after those genes were removed (Supplementary Section 11 and Supplementary Table S5). After removal of members of ARC and NMDAR sets, none of the subcategories comprising the synapse was significantly enriched 


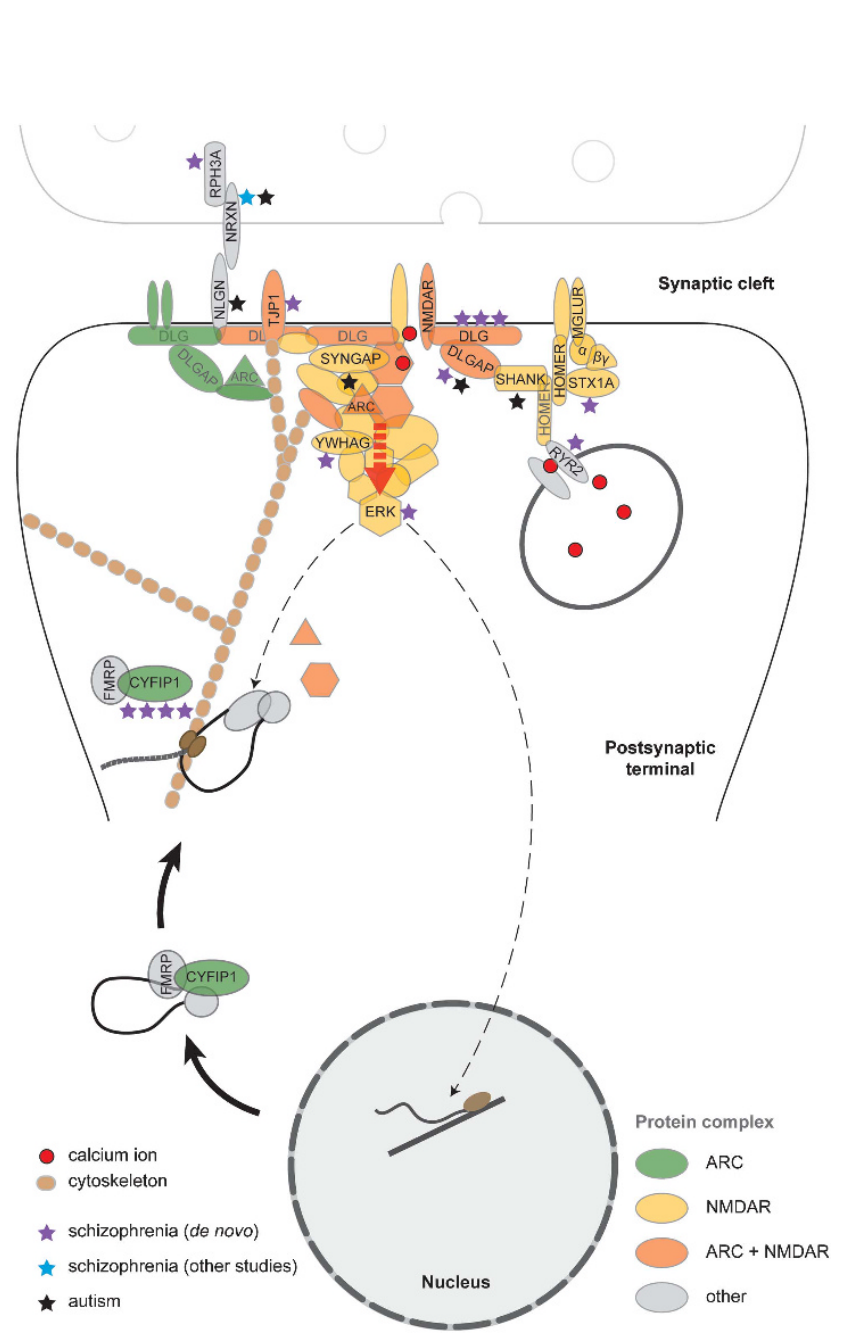

Figure 3 Disruption of postsynaptic signalling within activity-regulated cytoskeleton-associated protein (ARC) and $N$-methyl-D-aspartate receptor (NMDAR) complexes by copy number variants (CNVs). ARC and NMDAR bind to diverse structural and signalling molecules forming multiprotein complexes. Functional pathways encoded by these complexes are disrupted by de novo CNVs at multiple levels, as indicated by the purple asterisks (number of asterisks = number of de novos overlapping a gene or gene family). Calcium influx via the NMDAR, modulated by calcium release from internal stores (RYR2), drives downstream pathways whose association with the receptor is mediated by scaffold proteins (DLG1, DLG2, DLGAP1). Multiple pathways converge on ERK kinases (extracellular signal-regulated kinases), a focal point in the regulation of ARC transcription, dendritic localisation and local translation. ${ }^{47}$ ARC mRNA is transported to sites of synaptic activity in complexes containing CYFIP1, dissociation of which is required for ARC translation. ${ }^{49}$ CYFIP1 also regulates translation of CAMKII, ${ }^{49}$ a key component of NMDAR complexes. Although not identified in this study, deletions of synaptic adhesion protein NRXN1 (blue asterisk) have previously been found in schizophrenia. ${ }^{29} \mathrm{CNV}$ s disrupting genes within these same functional pathways have also been identified in autism ${ }^{30}$ (black asterisks).

except for 'synaptic vesicle membrane' $\left(P=4.22 \times 10^{-4}\right)$. These findings suggest enrichments in the PSD, and the great majority of that in the synapse GO gene set, is because of the enrichments in

ARC and NMDAR, but that there is residual enrichment elsewhere in the synapse that is captured by ‘synaptic vesicle membrane' genes (GO: 30672).

To exclude unknown possible sources of confounding arising from the use of control CNVs, we also compared gene sets hit by de novo CNVs from cases with those hit in random assignments of gene-hitting CNVs of the same size, ensuring the probability of a gene being hit was proportional to its size. Again, we observed significant enrichment of PSD genes $(P=0.0024)$, and a highly significant enrichment of the ARC $\left(2.21 \times 10^{-8}\right)$ and NMDAR $\left(2.95 \times 10^{-4}\right)$ complexes (Supplementary Section 12).

To exclude the possibility that our results reflect general properties of de novo CNVs, we compared case de novo CNVs with de novo CNVs identified in the control individuals from Iceland and from the Autism study by Sanders et al. ${ }^{25}$ Despite fewer control CNVs in these samples $(N=59$ and $N=14)$, and therefore reduced power, the findings were consistent with our primary analysis in showing significant enrichment of ARC and NMDAR (Table 2) as were those of sensitivity analysis restricted to very large CNVs $(>500 \mathrm{~kb}) \quad\left(\right.$ ARC $P=1.27 \times 10^{-4}$; NMDAR $\left.P=1.72 \times 10^{-2}\right)$.

Finally, we examined the ARC/NMDAR gene sets in the large case-control data sets. In this completely independent analysis, case CNVs were significantly enriched for members of the NMDAR $(P=0.0015)$ but not ARC complexes $(P=0.14)$. We note that in the de novo analysis, much of the additional signal for the ARC complex (over and above that of the NMDAR) comes from CNVs at 15q11.2 that span CYFIP1. Although there is strong published evidence for deletions at this locus being relevant to schizophrenia, ${ }^{3,7}$ this locus was not significantly enriched in the MGS study, ${ }^{4}$ and was excluded by the filtering criteria adopted by the ISC, ${ }^{2}$ the two studies that combined comprise a large proportion of the case-control data set we use in this study.

\section{Discussion}

Aiming to identify novel candidate CNV loci for schizophrenia, and to illuminate aspects of the pathophysiology of the disorder through gene-set enrichment analyses, we have conducted the largest analysis of de novo CNVs in schizophrenia to date. Although not every observed case de novo CNV is likely to be pathogenic, the hypothesis that a substantial proportion of them are likely to be so is supported by several observations. First, eight of the de novos occurred at already known schizophrenia CNV loci (Table 1, marked with footnote 'a'). Second, even after conservatively excluding those known loci, CNVs at the loci affected by case de novos occurred twice as frequently in cases in a meta-analysis of the largest available case-control CNV data sets. This elevation is much higher than the overall increase in CNV burden in cases in the large published studies. ${ }^{2,4}$ Third, in the trios sample, the rate of de novo CNVs 
was more than twice that observed in other control samples (Supplementary Table S3), suggesting that at least $50 \%$ of the case de novos are relevant to the pathophysiology of schizophrenia.

Our estimate of the de novo rate is lower than initial reports in autism ${ }^{23}$ and schizophrenia ${ }^{24}$ but is comparable with more recent estimates in autism. ${ }^{25,29,30}$ Post hoc evaluation suggests it is unlikely that our filtering steps excluded large numbers of true de novo CNVs within our target size range. Of the 34 Agilentvalidated de novos, 91\% $(N=31)$ had been rated (using MeZOD) as highly suggestive, whereas only $9 \%(N=3)$ had been called as ambiguous. Conversely, none of 33 putative de novos called by the Genotyping Console that were rejected by MeZOD were confirmed by Agilent.

It is notable that two previously documented schizophrenia loci, at $15 q 11.2$ and 15q13.3, were each found more than once as de novos (Table 1). Two other loci were represented by two de novo CNVs each: EHMT1 (encoding Eu-HMTase1), a histone H3 Lys 9 (H3-K9) methyltransferase, and DLG2 (encoding discs, large homologue 2). Moreover, one de novo spanned each of the related genes DLG1 (whose orthologue in Drosophila is also dlg1) and DLGAP1 (encoding discs large associated protein 1).

At EHMT1 we observed a total of two de novos, three additional exonic CNVs in cases and one in a control. EHMT1 haploinsufficieny has been implicated as the cause of the $9 q$ subtelomeric deletion syndrome (9qSTDS) characterised by moderate-tosevere mental retardation, childhood hypotonia and facial dysmorphisms, as well as a high prevalence of psychiatric symptoms in adulthood. ${ }^{31}$ A recent study has also reported strong evidence that deletions at EHMT1 are highly penetrant for phenotypes comprising developmental delay and a range of congenital anomalies. $^{32}$ With this additional evidence for the involvement of this gene in neurodevelopmental phenotypes, our data point to EHMT1 as a schizophrenia susceptibility gene. Intriguingly, in Drosophila, ehmt coordinates epigenetic changes important in regulating cognition. ${ }^{33}$ Our findings at this locus thus suggest a role for epigenetic mechanisms in at least some cases of schizophrenia, and potentially point the way to novel therapeutic opportunities as the developmental effects of ehmt mutation on cognition are reversible. ${ }^{31}$

The DLG (discs large) family of membrane-associated guanylate kinases (MAGUKs), which were hit by multiple case de novo CNVs, are components of postsynaptic signalling complexes that are embedded within the larger group of over 1000 proteins that make up the PSD. ${ }^{34}$ They are associated with NMDA receptors and are highly concentrated in synapses. Remarkably, the orthologue of DLG2 in Drosophila (dlg1) is directly regulated by the orthologue of EHMT1 (emht also known as G9a). ${ }^{33}$ CNVs spanning DLG1 and DLG2 have been reported before in schizophrenia ${ }^{4,6,17}$ whereas other members of the family (DLG3 and DLGAP2) have been implicated in mental retardation ${ }^{35}$ and autism..$^{30,36}$ Together with our observation of multiple de novos spanning members of this family, and the nominally significant association of exonic CNVs at DLG2 in the casecontrol analysis, the findings strongly suggest that the CNVs we report in $D L G$-related genes are likely to be of pathogenic relevance to schizophrenia.

Although not strongly implicated by our study, a number of singleton de novo CNVs are also of note as they are at loci known to be associated with rare genomic disorders. The first is a deletion at 1q21.1 reported in TAR syndrome ${ }^{37}$ (which does not overlap the known 1q21.1 schizophrenia locus ${ }^{2,7}$ ). We found the TAR region deleted in three more cases and only one control from the extended case-control samples. Again, deletions at this locus have very recently been strongly implicated in developmental delay. ${ }^{32}$ Another region is a duplication at the locus causing the $7 q 11.23$ microduplication syndrome (which is deleted in Williams-Beuren Syndrome), the prominent features of which include autism and developmental delay. ${ }^{38}$ Duplications at the WBS region were found in three more cases and one control. Although this excess is not statistically significant $(P=0.11$, uncorrected), given duplications at this locus have also recently been identified increasing susceptibility for autism ${ }^{25}$ and developmental delay, ${ }^{32}$ it seems likely that the observations in the present study point to the involvement of this locus in schizophrenia as well. Further details about each of these loci are provided in Supplementary Section 6.

Given that our data suggest de novo CNVs are highly enriched for pathogenic loci, we sought evidence for convergence of de novo events onto specific biological pathways using a hypothesis-led, systems biology approach. Many of the CNVs robustly implicated in schizophrenia are also implicated in neurodevelopmental disorders in which cognitive impairment is common. ${ }^{39-41}$ Moreover, as discussed in the Introduction, it has been hypothesised that schizophrenia CNVs are enriched for genes encoding proteins associated with synaptic function. Our findings of apparent convergence of de novo CNVs onto genes encoding MAGUK proteins broadly support this synaptic hypothesis and suggested that more refined examination of synaptic genes is warranted.

Cognitive deficits are increasingly recognised as core features of schizophrenia, and it has long been known that antagonism of NMDA receptors at glutamatergic synapses can induce a schizophrenialike psychosis that includes some of those deficits. ${ }^{42}$ This has led to a glutamate hypofunction hypothesis of schizophrenia. Glutamate receptors form multiprotein complexes with large sets of scaffold and signalling proteins including $\mathrm{MAGUKs}^{43}$ that are embedded in the PSD. It is clear that disruption of a number of synaptic proteins linked to glutamate receptor signalling alters cognitive function in rodents. ${ }^{44}$ The composition of the PSD has recently been identified in humans by some of the present authors, ${ }^{34}$ affording us an unprecedented opportunity 
to investigate the role of this complex in schizophrenia. Specifically, we tested the hypothesis that de novo CNVs in cases are enriched for genes encoding members of this complex.

We first compared the case de novos with a set of control CNVs drawn from the same population as the trios. Although those CNVs must have originally occurred as de novo mutations, predominantly transmitted CNVs clearly have different characteristics from the case de novos, most obviously size. Although our set-based analyses allow for size differences, to ensure our findings were robust to the control data set, we also compared gene sets hit by case de novo CNVs with those hit in random assignments of genehitting CNVs of the same size and obtained very similar gene-set enrichments. Finally, in order to exclude the possibility that our findings reflected general properties of de novo CNVs, we compared case de novo CNVs to two sets of control de novos, one drawn from the Icelandic population and the other from a much smaller sample of unaffected sibs of people with autism. Despite the wide disparities in the sources of the control CNVs, and the potential for different sources of bias, the results converge in pointing to the involvement of the synapse, the PSD, and more specifically, ARC and NMDAR complexes. Finally, and fully independent of those analyses, we show a significant enrichment for genes in the NMDAR complex in a meta-analysis of case-control data sets. We think it likely that the weaker finding for the NMDAR complex in the large case-control study compared with the relatively small de novo study, and the absence of association to ARC in the former, reflects the much lower power of the case-control design as a result of poorer enrichment for pathogenic CNVs.

This study adds to an accumulating body of evidence from human and animal genetic studies implicating disruption of synaptic processes in schizophrenia. ${ }^{45}$ By identifying an unprecedentedly large number of de novo CNVs in schizophrenia and demonstrating that these are likely to be highly enriched for pathogenic events, we have added substantially to the evidence implicating synaptic processes in schizophrenia. As well as implicating a set of functionally related synaptic proteins (EHMT1, $D L G 2, D L G 1$ and DLGAP1) we have identified a sufficient number of schizophrenia-enriched loci to identify potential points on convergence on specific synaptic complexes. Using gene sets that have been systematically annotated from individual, highquality proteomic data sets and multiple analytic approaches carefully controlled for biases, we not only provide strong evidence for the importance of synaptic proteins, but also provide novel convergent support for the involvement of NMDAR, and to a lesser extent ARC protein, complexes in the aetiology and pathogenesis of the disorder, both of which are involved in NMDA signal transduction. NMDA receptor signalling regulates induction of multiple forms of synaptic plasticity, ${ }^{46}$ with local synthesis of
ARC central to synaptic remodelling and the longterm maintenance of synaptic changes. ${ }^{47}$ Our finding that 12 out of the 34 case de novos impact on ARC and/or NMDAR complexes, supported by robust statistical analyses, suggest that disruption of NMDA signalling plays a key role in at least some cases of schizophrenia. As noted above, our findings do not exclude a role for mutations in other post- or presynaptic complexes, and given the close functional relationship between different synaptic components, we might expect pathology at a number of different points to play a role. Indeed, the robust association between NRXN1 deletions and schizophrenia ${ }^{48}$ points to presynaptic disruption in some cases, a hypothesis further supported by enrichment for case de novos in the GO category 'synaptic vesicle membrane' after adjustment for ARC and NMDAR. Our findings delineate a circumscribed set of largely postsynaptic proteins and functions that warrant further functional analysis in model systems.

\section{Conflict of interest}

The authors declare no conflict of interest.

\section{Acknowledgments}

We acknowledge the families who participated in the study. Funding for recruitment was provided by the Janssen Research Foundation. Genotyping was funded by multiple grants to the Stanley Center for Psychiatric Research at the Broad Institute from the Stanley Medical Research Institute, The Merck Genome Research Foundation and the Herman Foundation. Work at Cardiff University was funded by Medical Research Council (MRC) Programme (Ref G0800509) and Centre Grants, the National Institutes of Mental Health (USA) (CONTE: 2 P50 MH066392-05A1) and a grant from the EU (EU-GEI). Genotyping and CNV analysis in the Icelandic trios by deCODE Genetics was funded in part by EU grants: HEALTH-2007-2.2.1-10-223423 (Project PsychCNV), PIAG-GA-2008-218251 (Project PsychGene) and IMI-JU-NewMeds. SGNG, ÀB，EF，LNVL，JC，NHK and MOC were supported by the Genes to Cognition Program funded by the Wellcome Trust, MRC Project (Ref G06706B), EU grants: (Project GENCODYS Nr 241995, Project EUROSPIN No. 242498 and Project SYNSYS No. 242167). ÀB is supported by EMBO and the European Commission. We acknowledge Edward Scolnick for helpful discussions.

The following psychiatrists in Bulgaria recruited families for the project:

District Hospital Blagoevgrad: Nikolay Ianakiev, Radoi Milev, Valentina Kovachka.

University Hospital Sofia: Vihra Milanova, Georgy Koichev, Stefan Krastev, Georgi Onchev, Elitza Djatova, Tsveteslava Galabova, Desislava Ivanova, Ivan Gerdjikov, Emil Kaludiev, Christian Kostov, Ivanka Krastnikova, Irina Lazarova, Nedialko Mihailov, Maria Nikolova, Iliana Pristavova, Kalina 
Shoilekova, Anton Slavchev, Vladimir Sotirov, Svetla Stambolova, Miroslava Stamenova, Vessela Stoianova, Milena Strashimirova, Snezhina Tacheva, Todor Todorov, Rositza Vladimirova.

Plovdiv University Hospital: Marin Roglev, Inko Kehaiov, Nadezhda Poryazova, Rayna Tsvetkova, Petia Blagoeva, Veselina Vlahova.

Radnevo Psychiatric Hospital: Todor Tolev, Kalinka Drebova, Penka Grozeva, Ivan Ivanov, Mariya Nikolova, Maya Pashova, Radka Patricheva, Minka Sokolovska, Antoniya Terzieva, Donka Terzieva, Vania Tesheva, Ilina Topcheva, Vladimir Trynov.

Psychiatric Hospital, Russe: Temenujka Mateva, Biliana Mechkunova, Plamen Panayotov, Rositsa Vacheva.

Psychiatric Dispensary, Sofia: Lubomir Jivkov, Svetlinka Koleva, Kalina Georgieva, Tatiana Kanova, Anna Maikova, Krasimira Marcheva, Vesselin Sekoulov, Diana Shkodrova, Vesselina Slavcheva, Tenko Tenev, Lora Tsoneva.

DNA handling and extraction was performed at the Department of Medical Genetics, University Hospital 'Maichin Dom', Sofia (Head of Department Professor Draga Toncheva): Lyudmila Georgieva, Albena Dimitrova and Irina Zaharieva.

\section{Author Contributions}

GK, MJO, MCO’D, PS and SP designed the study. GK, MJO and MCO'D drafted the primary manuscript along with AJP, PH and SG. The primary genotyping analysis with Affymetrix arrays was organised and coordinated by SP, PS, JM and KC. MI, GK, DR, SP, MF, DI and RW analysed the raw intensity data. CNV analysis was performed by GK, DI, MF, ER and DR. MF and GK designed the Agilent custom arrays. DG, MI, LG and GK performed the Agilent validation experiments. HS, KS, PIO and YB were responsible for analysis of Icelandic trios. Synapse proteomic data sets were prepared by LNVL, ÀB, EF, MOC, JC and SGNG. AJP and SGNG identified the curated sets of genes for pathway analyses. $\mathrm{PH}$ and AJP analysed the involvement of gene sets in de novo CNVs. SP undertook an independent parallel analysis. GK and MJO planned and organised the recruitment of families, which was coordinated by GK, IN and DT. DI wrote the scripts used for de novo analysis, including the $z$-score method. All authors discussed the results, their interpretation and contributed to the final manuscript.

\section{References}

1 Owen MJ, Craddock N, O’Donovan MC. Suggestion of roles for both common and rare risk variants in genome-wide studies of schizophrenia. Arch Gen Psychiatry 2010; 67: 667-673.

2 International Schizophrenia Consortium (ISC). Rare chromosomal deletions and duplications increase risk of schizophrenia. Nature 2008; 455: 237-241.

3 Kirov G, Grozeva D, Norton N, Ivanov D, Mantripragada KK, Holmans $\mathrm{P}$ et al. Support for the involvement of large CNVs in the pathogenesis of schizophrenia. Hum Mol Genet 2009; 18: 1497-1503.
4 Levinson DF, Duan J, Oh S, Wang K, Sanders AR, Shi J et al. Copy number variants in schizophrenia: confirmation of five previous findings and new evidence for 3q29 microdeletions and VIPR2 duplications. Am J Psychiatry 2011; 168: 302-316.

5 Moreno-De-Luca D, Mulle JG, Kaminsky EB, Sanders SJ, Myers SM, Adam MP et al. Deletion 17q12 is a recurrent copy number variant that confers high risk of autism and schizophrenia. Am J Hum Genet 2010; 87: 618-630.

6 Mulle JG, Dodd AF, McGrath JA, Wolyniec PS, Mitchell AA, Shetty AC et al. Microdeletions of 3q29 confer high risk for schizophrenia. Am J Hum Genet 2010; 87: 229-236.

7 Stefansson H, Rujescu D, Cichon S, Pietilainen OPH, Ingason A, Steinberg $\mathrm{S}$ et al. Large recurrent microdeletions associated with schizophrenia. Nature 2008; 455: 232-236.

8 Vacic V, McCarthy S, Malhotra D, Murray F, Chou H-H, Peoples A et al. Duplications of the neuropeptide receptor gene VIPR2 confer significant risk for schizophrenia. Nature 2011; 471: 499-503.

9 Ingason A, Kirov G, Giegling I, Hansen T, Isles AR, Jakobsen KD et al. Maternally derived microduplications at 15q11-q13: implication of imprinted genes in psychotic illness. Am J Psychiatry 2011; 168: 408-417.

10 Ingason A, Rujescu D, Cichon S, Sigurdsson E, Sigmundsson T, Pietilainen $\mathrm{OPH}$ et al. Copy number variations of chromosome 16p13.1 region associated with schizophrenia. Mol Psychiatry 2011; 16: $17-25$.

11 Kirov G, Rujescu D, Ingason A, Collier DA, O’Donovan MC, Owen MJ. Neurexin 1 (NRXN1) deletions in schizophrenia. Schizophr Bull 2009; 35: 851-854.

12 McCarthy SE, Makarov V, Kirov G, Addington AM, McClellan J, Yoon S et al. Microduplications of 16p11.2 are associated with schizophrenia. Nat Genet 2009; 41: 1223-1227.

13 Sebat J, Levy DL, McCarthy SE. Rare structural variants in schizophrenia: one disorder, multiple mutations; one mutation, multiple disorders. Trends Genet 2009; 25: 528-535.

14 Grozeva D, Kirov G, Ivanov D, Jones IR, Jones L, Green EK et al. Rare copy number variants: a point of rarity in genetic risk for bipolar disorder and schizophrenia. Arch Gen Psychiatry 2010; 67: 318-327.

15 Kirov G, Gumus D, Chen W, Norton N, Georgieva L, Sari M et al. Comparative genome hybridization suggests a role for NRXN1 and APBA2 in schizophrenia. Hum Mol Genet 2008; 17: 458-465.

16 Tam GWC, Redon R, Carter NP, Grant SGN. The role of DNA copy number variation in schizophrenia. Biol Psychiatry 2009; 66: 1005-1012.

17 Walsh T, McClellan JM, McCarthy SE, Addington AM, Pierce SB, Cooper GM et al. Rare structural variants disrupt multiple genes in neurodevelopmental pathways in schizophrenia. Science 2008; 320: 539-543.

18 Glessner JT, Reilly MP, Kim CE, Takahashi N, Albano A, Hou C et al. Strong synaptic transmission impact by copy number variations in schizophrenia. Proc Natl Acad Sci USA 2010; 107: 10584-10589.

19 Raychaudhuri S, Korn JM, McCarroll SA, Consortium TIS, Altshuler D, Sklar P et al. Accurately assessing the risk of schizophrenia conferred by rare copy-number variation affecting genes with brain function. PLoS Genet 2010; 6: pii: e1001097.

20 Bundy H, Stahl D, MacCabe JH. A systematic review and metaanalysis of the fertility of patients with schizophrenia and their unaffected relatives. Acta Psychiat Scand 2010; 123: 98-106.

21 Laursen TM, Munk-Olsen T. Reproductive patterns in psychotic patients. Schizophr Res 2010; 121: 234-240.

22 Rees E, Moskvina V, Owen MJ, O’Donovan MC, Kirov G. De novo rates and selection of schizophrenia-associated copy number variants. Biol Psychiatry; advance online publication, 18 August 2011 [e-pub ahead of print].

23 Sebat J, Lakshmi B, Malhotra D, Troge J, Lese-Martin C, Walsh T et al. Strong association of de novo copy number mutations with autism. Science 2007; 316: 445-449.

24 Xu B, Roos JL, Levy S, van Rensburg EJ, Gogos JA, Karayiorgou M. Strong association of de novo copy number mutations with sporadic schizophrenia. Nat Genet 2008; 40: 880-885.

25 Sanders Stephan J, Ercan-Sencicek AG, Hus V, Luo R, Murtha Michael T, Moreno-De-Luca D et al. Multiple recurrent de novo CNVs, including duplications of the 7q11.23 Williams 
syndrome region, are strongly associated with autism. Neuron 2011; 70: 863-885.

26 Ikeda M, Aleksic B, Kirov G, Kinoshita Y, Yamanouchi Y, Kitajima $\mathrm{T}$ et al. Copy number variation in schizophrenia in the Japanese population. Biol Psychiatry 2010; 67: 283-286.

27 Wang K, Li M, Hadley D, Liu R, Glessner J, Grant SFA et al. PennCNV: an integrated hidden Markov model designed for high-resolution copy number variation detection in whole-genome SNP genotyping data. Genome Res 2007; 17: 1665-1674.

28 Pinto D, Darvishi K, Shi X, Rajan D, Rigler D, Fitzgerald T et al. Comprehensive assessment of array-based platforms and calling algorithms for detection of copy number variants. Nat Biotech 2011; 29: 512-520.

29 Rujescu D, Ingason A, Cichon S, Pietiläinen OP, Barnes MR Toulopoulou $\mathrm{T}$ et al. Disruption of the neurexin 1 gene is associated with schizophrenia. Hum Mol Genet 2009; 18: 988-996.

30 Pinto D, Pagnamenta AT, Klei L, Anney R, Merico D, Regan R et al. Functional impact of global rare copy number variation in autism spectrum disorders. Nature 2010; 466: 368-372.

31 Kleefstra T, van Zelst-Stams WA, Nillesen WM, Cormier-Daire V, Houge G, Foulds $\mathrm{N}$ et al. Further clinical and molecular delineation of the $9 \mathrm{q}$ subtelomeric deletion syndrome supports a major contribution of EHMT1 haploinsufficiency to the core phenotype. J Med Genet 2009; 46: 598-606.

32 Cooper GM, Coe BP, Girirajan S, Rosenfeld JA, Vu TH, Baker C et al. A copy number variation morbidity map of developmental delay. Nat Genet 2011; 43: 838-846.

33 Kramer JM, Kochinke K, Oortveld MAW, Marks H, Kramer D, de Jong EK et al. Epigenetic regulation of learning and memory by Drosophila EHMT/G9a. PLoS Biol 2011; 9: e1000569.

34 Bayés A, van de Lagemaat LN, Collins MO, Croning MDR, Whittle IR, Choudhary JS et al. Characterization of the proteome, diseases and evolution of the human postsynaptic density. Nat Neurosci 2011; 14: 19-21.

35 Tarpey P, Parnau J, Blow M, Woffendin H, Bignell G, Cox C et al. Mutations in the DLG3 gene cause nonsyndromic X-linked mental retardation. Am J Hum Genet 2004; 75: 318-324.

36 Guilmatre A, Dubourg C, Mosca A-L, Legallic S, Goldenberg A, Drouin-Garraud V et al. Recurrent rearrangements in synaptic and neurodevelopmental genes and shared biologic pathways in schizophrenia, autism, and mental retardation. Arch Gen Psychiatry 2009; 66: 947-956.

37 Klopocki E, Schulze H, Strau G, Ott C-E, Hall J, Trotier F et al. Complex inheritance pattern resembling autosomal recessive inheritance involving a microdeletion in thrombocytopenia-absent radius syndrome. Am J Hum Genet 2007; 80: 232-240.

38 Van der Aa N, Rooms L, Vandeweyer G, van den Ende J, Reyniers E, Fichera $\mathrm{M}$ et al. Fourteen new cases contribute to the characterization of the 7q11.23 microduplication syndrome. Eur J Med Genet 2009; 52: 94-100.

39 McCarthy SE, Makarov V, Kirov G, Addington AM, McClellan J, Yoon $\mathrm{S}$ et al. Microduplications of $16 \mathrm{p} 11.2$ are associated with schizophrenia. Nat Genet 2009; 41: 1223-1227.

40 Sebat J, Levy DL, McCarthy SE. Rare structural variants in schizophrenia: one disorder, multiple mutations; one mutation, multiple disorders. Trends Genet 2009; 25: 528-535.

41 Williams NM, Zaharieva I, Martin A, Langley K, Mantripragada K, Fossdal $\mathrm{R}$ et al. Rare chromosomal deletions and duplications in attention-deficit hyperactivity disorder: a genome-wide analysis. Lancet 2010; 376: 1401-1408.

42 Abi-Saab WM, D’Souza DC, Moghaddam B, Krystal JH. The NMDA antagonist model for schizophrenia: promise and pitfalls. Pharmacopsychiatry 1998; 31(S 2): 104-109.

43 Husi H, Ward MA, Choudhary JS, Blackstock WP, Grant SGN Proteomic analysis of NMDA receptor-adhesion protein signaling complexes. Nat Neurosci 2000; 3: 661-669.

44 Grant SG, Marshall MC, Page KL, Cumiskey MA, Armstrong JD Synapse proteomics of multiprotein complexes: en route from genes to nervous system diseases. Hum Mol Genet 2005 14: R225-R234.

45 Mitchell KJ. The genetics of neurodevelopmental disease. Cur Opin Neurobiol 2011; 21: 197-203.

46 Malenka RC, Nicoll RA. NMDA-receptor-dependent synaptic plasticity: multiple forms and mechanisms. Trends Neurosci 1993; 16: 521-527.

47 Bramham C, Alme M, Bittins M, Kuipers S, Nair R, Pai B et al The Arc of synaptic memory. Exp Brain Res 2010; 200: 125-140.

48 Kirov G. The role of copy number variation in schizophrenia. Exp Rev Neurotherapeutics 2010; 10: 25-32.

49 Napoli I, Mercaldo V, Boyl PP, Eleuteri B, Zalfa F, De Rubeis S et al. The fragile $\mathrm{X}$ syndrome protein represses activity-dependent translation through CYFIP1, a new 4E-BP. Cell 2008; 134: 1042-1054.

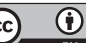

This work is licensed under a Creative Commons Attribution 3.0 Unported License. To view a copy of this license, visit http:// creativecommons.org/licenses/by/3.0/

Supplementary Information accompanies the paper on the Molecular Psychiatry website (http://www.nature.com/mp) 